\title{
Evaluation of the Dwell-Time and Dose Difference in Intravesical Bacillus Calmette-Guèrin Therapy
}

\author{
Takashi Nagai $^{1}$, Takehiko Okamura ${ }^{1 *}$, Yutaro Tanaka ${ }^{1,2}$, Yoshinobu Moritoki ${ }^{1}$, \\ Daichi Kobayashi $^{1}$, Takahiro Kobayashi ${ }^{1}$, Hidetoshi Akita ${ }^{1}$, Takahiro Yasui ${ }^{2}$
}

\begin{abstract}
Objective: Bacillus Calmette-Guèrin (BCG) intravesical therapy is currently established using a low dose because of the high incidence of side-effects. Moreover, shortening the dwell time of BCG is conducted in some facilities owing to the complications associated with a long dwell time after injection. The method of BCG administration varies in each facility and even with each doctor. We evaluated whether the dwell-time and dose differences in patients who underwent intravesical BCG therapy is related to completion rates, adverse effects, and nonrecurrence rates. Methods: From November 2006 to April 2016, a total of 173 patients who received intravesical BCG therapy after transurethral resection of bladder tumor or transurethral biopsy were evaluated retrospectively. We allocated them into 4 groups based on the dose (40 or $80 \mathrm{mg} \mathrm{BCG)} \mathrm{and} \mathrm{the} \mathrm{dwell} \mathrm{time} \mathrm{(} 1$ or 2 hours). Completion rate, side effects, and nonrecurrence rates were evaluated. Results: No significant improvement in the completion rate or reduction in side-effects was observed in any of the regimens. Although nonrecurrence rates for the 1-hour dwell time tended to be lower than the 2-hour dwell time, the difference was not significant. Conclusion: Our study suggests that reducing the BCG dose or shortening the dwell time does not reduce adverse effects or affect the nonrecurrence rate.
\end{abstract}

Keywords: BCG- dwell-time difference- dose difference

Asian Pac J Cancer Prev, 20 (5), 1389-1392

\section{Introduction}

Bacillus Calmette-Guèrin (BCG) intravesical therapy, now used widely all over the world (Sylvester et al., 2002; Schwaibold et al., 2006), is recommended as adjuvant prophylactic therapy for high risk non-muscle invasive bladder cancer in the American Urological Association guidelines (Chang et al., 2016). However, because of the high incidence of a variety of systemic side-effects associated with BCG intravesical therapy, a low-dose administration option is currently established, including subsequent maintenance treatment (Lockyer et al., 2002; Andius et al., 2005). In addition, shortening the BCG dwell time is used to reduce complications associated after intravesical injection (Martínez-Piñeiro et al., 2002; Zeng et al., 2015; Yokomizo et al., 2016).

The method of BCG administration varies in each facility and even by individual doctors. We retrospectively evaluated different dose and dwell-time regimens in our hospital to determine if either parameter is related to completion rates, adverse effects, and nonrecurrence rates in patients receiving intravesical BCG therapy.

\section{Materials and Methods}

We retrospectively evaluated 173 patients who received $\mathrm{BCG}$ therapy following transurethral resection of bladder tumor (TURBT) or transurethral (TU) biopsy from November 2006 to April 2016. Patients received 80 or $40 \mathrm{mg}$ of BCG (Tokyo strain, Japan BCG Laboratory, Tokyo, Japan) as induction treatment. This included two multi-institutional joint research programs were on-going and the patients who participated in these studies had a 2-hour dwell time. The 173 patients were categorized into 4 groups based on the BCG dose and the dwell time as follows: 76 patients received $80 \mathrm{mg}$ with a 1-hour dwell time (A1 group), 23 patients received $80 \mathrm{mg}$ BCG with a 2-hour dwell time (A2 group), 66 patients received 40 mg with a 1-hour dwell time (B1 group), and 8 patients received $40 \mathrm{mg}$ with a 2-hour dwell time (B2 group). BCG was administered once weekly for 6-8 consecutive weeks. Treatment response was evaluated by cystoscopy, TU biopsy, TURBT, and three urine cytology examinations 4-12 weeks after the final BCG instillation. Patients with bladder cancers with muscle invasion (more than stage pT2) were excluded from the present series, together with those undergoing previous urinary tract surgery,

${ }^{1}$ Department of Urology, Anjo Kosei Hospital, 28 Higashihirokute, Anjo-cho, Anjo, ${ }^{2}$ Department of Nephro-Urology, Nagoya City University, Graduate School of Medical Sciences, Kawasumi 1, Mizuho-cho, Mizuho-ku, Nagoya, Japan. *For Correspondence: hiko2546@sf.commufa.jp 
or given intravesical or general chemotherapy. Patients were asked to refrain, where possible, from urination within 2 hours of the instillation and were monitored for bladder irritation, temperature change, and other clinical symptoms. A tuberculin test, blood examinations, chest $\mathrm{X}$-rays, cystoscopy, and urinary cytology were conducted in all cases prior to BCG instillation and also at other times when considered appropriate. Follow up was performed once per week during the weekly treatment periods, and then every 1 to 3 months after cessation of treatment, depending on the patient's situation.

Recurrence with progression was defined with reference to muscle invasive disease (Sylvester et al., 2002; Schwaibold et al., 2006). Recurrence free survival was defined as the period between the last BCG induction instillation and the follow-up evaluation with no evidence of recurrence.

Surgically resected materials were routinely fixed in $10 \%$ buffered formalin and embedded in paraffin for sectioning and histopathological assessment of hematoxylin and eosin-stained sections. Tumor grading and staging were performed with reference to the $1 \mathrm{st}$ edition of the "General Rules for Clinical and Pathological Studies on Bladder Cancer of the Japanese Urological Association and the Japanese Society of Pathology " (The Japanese Urological Association, The Japanese Society of Pathology and Japan Radiological Society, 2011).

Nonrecurrence rates were estimated using the Kaplan-Meier method, and the significance of differences between curves was tested by the log-rank test. A value of $\mathrm{p}<0.05$ was considered statistically significant. Simple linear regression analysis was used to determine the correlation coefficient between the period of BCG treatment and clinical variables. All statistical analyses were performed using SPSS Version 17 (IBM, Armonk, NY, USA). The research was performed under approval of the institutional review board of Anjo Kosei Hospital (review board number: R16-021).

\section{Results}

Patient characteristics, including age, the ratio of male: female, tumor grade, $\mathrm{T}$ category, need for second TURBT before BCG, and number of tumors are shown in Table 1. The completion rates for each group were as follows: $67 / 76$ (88.2\%), 21/23 (91.3\%),54/66 (81.8\%) and 7/8(87.5\%) for groups A1, A2, B1, and B2, respectively. Adverse effects for each group are shown in Figure 1. There were no significant differences between the groups. A total of 24 patients could not complete the scheduled course. Of these cases, bladder irritation symptoms were seen in 5/24 $(20.8 \%)$, general malaise in $5 / 24(20.8 \%)$, fever in $3 / 24$ $(12.5 \%)$, Reiter's syndrome in $2 / 24(8.3 \%)$ and others in $9 / 24(37.5 \%)$. The nonrecurrence rates were as follows: $52 / 76$ (68.4\%), 19/23 (82.6\%), 46/66 (69.7\%), and 7/8 $(87.5 \%)$ for groups A1, A2, B1, and B2, respectively. The Kaplan-Meier analysis of nonrecurrence rates are shown in Figure 2. There were no significant differences by group (A1 vs B1, $\mathrm{p}=0.973$; $\mathrm{A} 1$ vs $\mathrm{A} 2, \mathrm{p}=0.385$; $\mathrm{A} 1$ vs B2, $\mathrm{p}=0.187$; 2 vs $\mathrm{B} 1, \mathrm{p}=0.322 ; \mathrm{A} 2$ vs $\mathrm{B} 2, \mathrm{p}=0.502$; and $\mathrm{B} 1$ vs $B 2, p=0.145)$. Moreover, we compared the groups based on dose (A1 and A2 vs B1 and B2) and dwell time (A1 and $\mathrm{B} 1$ vs $\mathrm{A} 2$ and $\mathrm{B} 2$ ). Figure 3 shows the Kaplan-Meier analysis of the nonrecurrence rates based on dose (Figure $3 \mathrm{~A}$ ) and dwell time (Figure 3B). The nonrecurrence rate was not significantly different between $80 \mathrm{mg}$ and $40 \mathrm{mg}$ $(\mathrm{p}=0.852)$. Although nonrecurrence rates for the 1-hour dwell time tended to be lower than the 2-hour dwell time, the difference was not significant $(\mathrm{p}=0.122)$.

Table 1. Patient and Tumor Characteristics. A1; 80mg, 1 hour, A2; 80mg, 2 hours, B1; 40mg, 1 hour, B2; 40mg, 2hours. TUR=transurethral resection, $\mathrm{CIS}=$ carcinoma in situ

\begin{tabular}{|c|c|c|c|c|}
\hline & A1 & A2 & B1 & B2 \\
\hline No. of patients & 76 & 23 & 66 & 8 \\
\hline Median age, years (range) & $70(44-82)$ & $65(39-79)$ & $80(53-89)$ & $72(54-80)$ \\
\hline Male: Female & $60: 16$ & $16: 7$ & $52: 14$ & $7: 1$ \\
\hline \multicolumn{5}{|l|}{ Grade, n (\%) } \\
\hline 1 & $3(3.9 \%)$ & $7(30.4 \%)$ & $6(9.1 \%)$ & $0(0 \%)$ \\
\hline 2 & $55(72.4 \%)$ & $11(47.8 \%)$ & $36(54.5 \%)$ & $5(62.5 \%)$ \\
\hline 3 & $18(23.7 \%)$ & $5(21.7 \%)$ & $24(36.4 \%)$ & $3(37.5 \%)$ \\
\hline \multicolumn{5}{|l|}{ T category, n (\%) } \\
\hline is & $10(13.2 \%)$ & $3(13.0 \%)$ & $16(24.2 \%)$ & $2(25.0 \%)$ \\
\hline $\mathrm{a}$ & $40(52.6 \%)$ & $15(65.2 \%)$ & $31(47.0 \%)$ & $2(25.0 \%)$ \\
\hline 1 & $26(34.2 \%)$ & $5(21.7 \%)$ & $19(28.8 \%)$ & $4(50.0 \%)$ \\
\hline 2nd TUR yes vs no & $13: 63$ & $3: 20$ & $5: 61$ & $0: 8$ \\
\hline \multicolumn{5}{|l|}{ No. of tumors, n (\%) } \\
\hline 1 & $10(13.2 \%)$ & $3(13.0 \%)$ & $9(13.6 \%)$ & $0(0 \%)$ \\
\hline $2-7$ & $41(53.9 \%)$ & $16(69.6 \%)$ & $33(50.0 \%)$ & $5(62.5 \%)$ \\
\hline$>8$ & $14(18.4 \%)$ & $1(4.3 \%)$ & $8(12.1 \%)$ & $1(12.5 \%)$ \\
\hline CIS & $11(14.5 \%)$ & $3(13.0 \%)$ & $16(24.2 \%)$ & $2(25.0 \%)$ \\
\hline
\end{tabular}




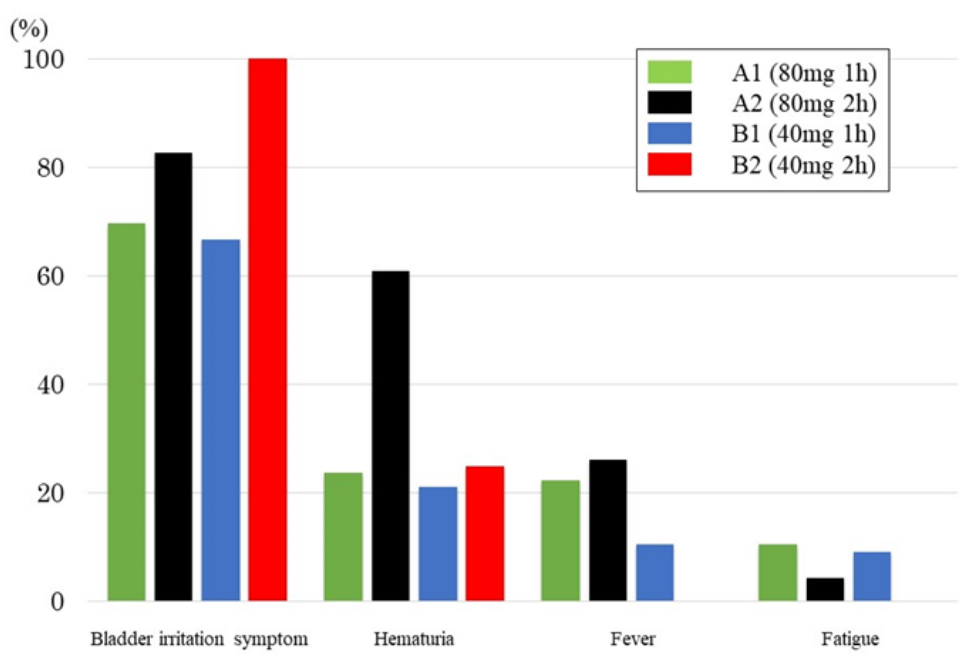

Figure 1. The Rates of Adverse Effects for Each Group. A1; 80mg, 1 hour, A2; 80mg, 2 hours, B1; 40mg, 1 hour, B2; $40 \mathrm{mg}$, 2hours

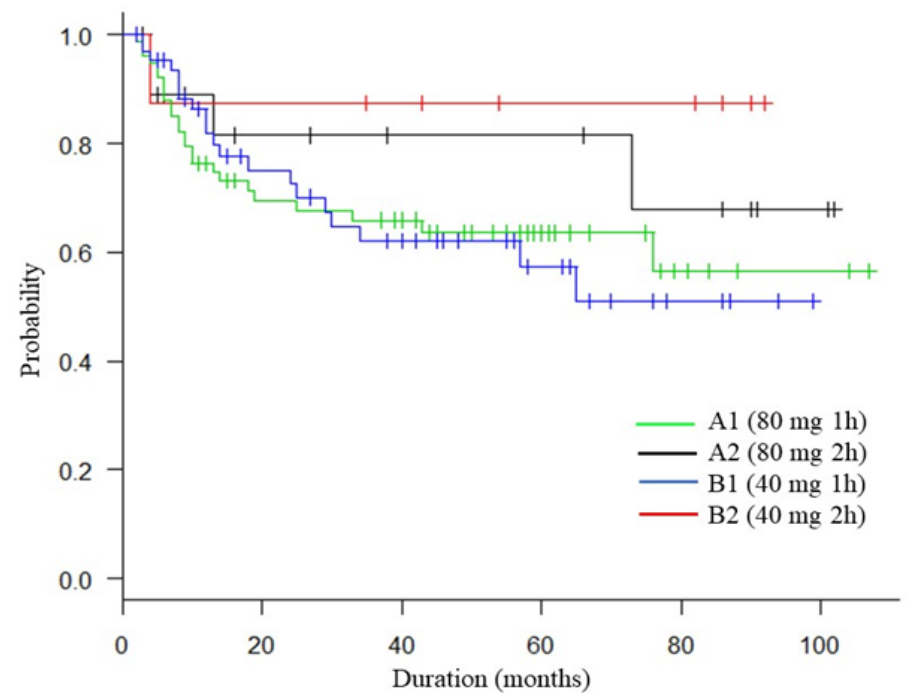

Figure 2. The Nonrecurrence Rates in Each Group. A1; 80mg, 1 hour, A2; 80mg, 2 hours, B1; 40mg, 1 hour, B2; $40 \mathrm{mg}$, 2hours

\section{Discussion}

Our study suggests that reducing the BCG dose or shortening the dwell time does not reduce adverse effects or affect the nonrecurrence rate.

In terms of dose reduction, Zeng et al., (2015) reported the efficacy of a reduced dose of BCG. They conducted a meta-analysis of randomized controlled
(A)

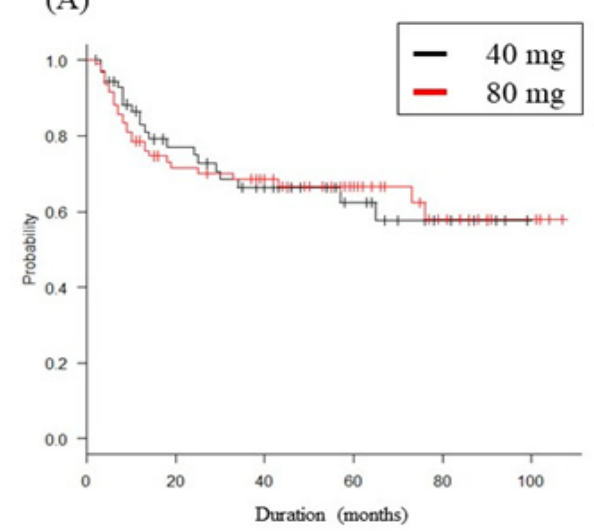

(B)

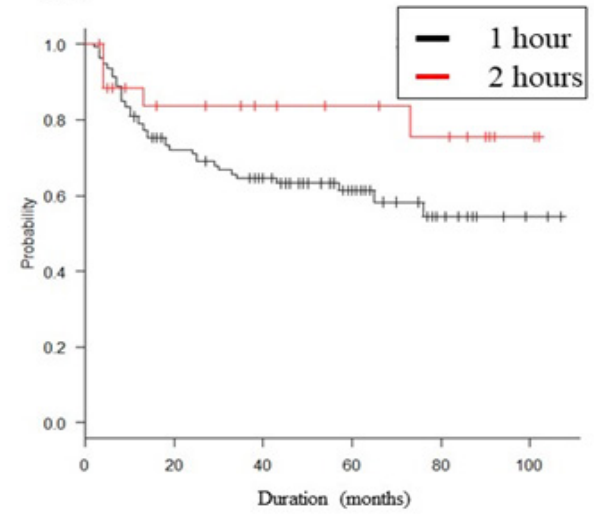

Figure 3. The Nonrecurrence Rates Based on Dose (A) or Dwell Time (B) 
trials and concluded that low-dose BCG instillation reduces the incidence of side effects while the oncological efficacy is not inferior to a standard dose. Yokomizo et al., (2016) also reported the advantage of dose reduction of BCG in terms of side effect in Japanese randomized controlled study. Martínez-Piñeiro et al., (2002) reported long-term follow-up of a randomized prospective trial that compared $81 \mathrm{mg}$ vs $27 \mathrm{mg}$ of intravesical BCG therapy and concluded that the lower dose had similar effectiveness with significantly less toxicity (Martínez-Piñeiro et al., 2002). Moreover, they recommended using a standard dose for high-risk tumors and a reduced dose for intermediate risk tumors or maintenance (Martínez-Piñeiro et al., 2002). On the other hand, Yalçinkaya et al. (1998) reported that an 81-mg dose was more effective for non-recurrence with less side effects compared with a 54-mg dose. Shah et al., (2016) reported that the effects of BCG on urothelial carcinoma cells demonstrate a direct dose-response relationship. In our study, there are no significant difference in side effects or nonrecurrence rate between 4 groups. It suggests that we might not need to care about the dose of BCG unless there are some particular reasons.

With regard to dwell time, Andius et al., (2005) reported that reducing dwell time to $\leq 30$ minutes could be alternative to a dose reduction to reduce pronounced adverse effects. Lockyer et al., (2002) reported a 1-hour dwell time BCG administration series, but they did not report adverse effects and also no description of reduced dose. From the viewpoint of safety, Drake et al., (1998) suggested that administration of BCG should be limited to 1 hour once per week for a maximum of 8 weeks. In our study, there are no significant difference of side effects and nonrecurrence rate, but in terms of nonrecurrence rate, 2 hours groups tend to be better prognosis than 1 hour groups. To bring better nonrecurrence rate, we might as well take this tendency into consideration in choosing dose as there are no significant difference in side effects. On the other hand, both of 2 hours group tend to be higher rate of bladder irritation symptom so that this is not a small problem that standing the symptom 1 more hour for out patients. We should select dwell time of BCG according to the condition of the patients.

This study has several limitations that must be taken into account while interpreting the results. First, this was a retrospective study with a small number of patients and there was variability among the 4 groups. Moreover, the assessment of therapy or decision of continuing treatment might have varied because patients were judged by different attending doctors. The precise dose reduction required for patients with adverse effects remains unclear when considering the effectiveness of the treatment. Further investigation is needed to analyze the appropriate dose and dwell time for BCG administration, including tailor-made, individualized options for patients based on their tolerance and response.

In conclusion, it has been reported that shortening the dwell time can reduce the side effects caused by BCG. However, in this study, no improvement in the completion rate or reduction in side effects was observed. The nonrecurrence rate was lower for the 1-hour dwell time compared with the standard 2-hour dwell time but there was no significant difference. Therefore, dose adjustment might be less prioritized and a 2-hour dwell time might as well be chosen over a 1-hour dwell time.

\section{References}

Andius P, Fehrling M, Holmäng S (2005). Intravesical bacillus Calmette-Guèrin therapy: experience with a reduced dwell-time in patients with pronounced side-effects. $B J U$ Int, 96, 1290-3.

Chang SS, Boorjian SA, Chou R, et al (2016). Diagnosis and treatment of non-muscle invasive bladder cancer: AUA/ SUO Guideline. J Urol, 196, 1021-9.

Drake MJ, Nixon PM, Crew JP (1998). Drug-induced bladder and urinary disorders. Incidence, prevention and management. Drug Saf, 19, 45-55.

Lockyer CR, Sedgwick JE, Gillatt DA (2002). Beware the BCG failures: a review of one institution's results. Eur Urol, 42, 542-6.

Martínez-Piñeiro JA, Flores N, Isorna S, et al (2002). Long-term follow-up of a randomized prospective trial comparing a standard $81 \mathrm{mg}$ dose of intravesical bacille Calmette-Guérin with a reduced dose of $27 \mathrm{mg}$ in superficial bladder cancer. BJU Int, 89, 671-80.

Schwaibold HE, Sivalingam S, May F, et al (2006). The value of a second transurethral resection for T1 bladder cancer. BJU Int, 97, 1199-1201.

Shah G, Zhang G, Chen F, et al (2016). The dose-response relationship of bacillus Calmette-Guérin and yrothelial carcinoma cell biology. J Urol, 195, 1903-10.

Sylvester RJ, van der Meijden AP, Lamm DL (2002). Intravesical bacillus Calmette-Guerin reduces the risk of progression in patients with superficial bladder cancer: a meta-analysis of the published results of randomized clinical trials. J Urol, 168, 1964-70.

The Japanese Urological Association, The Japanese Society of Pathology and Japan Radiological Society (2011). General Rule for Clinical and Pathological Studies on Bladder Cancer of Renal Pelvic, Ureteral and Bladder Cancer. (3rd edn), Kanehara Shuppan (Tokyo), 59-63.

Yalçinkaya F, Kamiş L, Ozteke O, et al (1998). Prospective randomized comparison of intravesical BCG therapy with standard dose versus low doses in superficial bladder cancer. Int Urol Nephrol, 30, 41-4

Yokomizo A, Kanimoto Y, Okamura T, et al (2016). Randomized controlled study of the efficacy, safety and quality of life with low dose bacillus Calmette-Guérin instillation therapy for nonmuscle invasive bladder cancer. J Urol, 195, 41-6.

Zeng S, Yu X, Ma C, et al (2015). Low-dose versus standard dose of bacillus Calmette-Guerin in the treatment of nonmuscle invasive bladder cancer: a systematic review and meta-analysis. Medicine (Baltimore), 94, e2176.

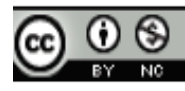

This work is licensed under a Creative Commons AttributionNon Commercial 4.0 International License. 